\title{
Canadian Rheumatology Association Recommendation for the Use of COVID-19 Vaccination for Patients With Autoimmune Rheumatic Diseases
}

\author{
Glen S. Hazlewood ${ }^{1}$ (D) Jordi Pardo Pardo ${ }^{2}$ Cheryl Barnabe $^{1}$ (D), Orit Schieir ${ }^{3}$ iD, Claire E.H. Barber ${ }^{1}$ (D), \\ Sasha Bernatsky ${ }^{4}$, Ines Colmegna ${ }^{5}$ (D), Carol Hitchon ${ }^{6}$ (D), Mark Loeb ${ }^{7}$, Dominik Mertz ${ }^{7}$ iD, \\ Laurie Proulx ${ }^{8}$ (D), Dawn P. Richards ${ }^{8}$ iD, Rosie Scuccimarri ${ }^{9}$ (D), Peter Tugwell ${ }^{10}$ (D), \\ Holger J. Schünemann ${ }^{7}$ (D), Reza D. Mirza ${ }^{11}$ (D), Alan L. Zhou ${ }^{10}$ iD, Roko P.A. Nikolic ${ }^{12}$ (D), \\ Megan Thomas ${ }^{13}$, Helena Chase ${ }^{14}$, Maede Ejaredar ${ }^{13}$, and Robby Nieuwlaat ${ }^{15}$
}

ABSTRACT. Objective. To develop guidance on the use of coronavirus disease 2019 (COVID-19) vaccines in patients with autoimmune rheumatic diseases (ARD).

Methods. The Canadian Rheumatology Association (CRA) formed a multidisciplinary panel including rheumatologists, researchers, methodologists, vaccine experts, and patients. The panel used the GRADE (Grading of Recommendations Assessment, Development, and Evaluation) approach. Outcomes were prioritized according to their importance for patients and clinicians. Evidence from the COVID-19 clinical trials was summarized. Indirect evidence for non-COVID-19 vaccines in ARD was also considered. The GRADE evidence-to-decision (EtD) framework was used to develop a recommendation for the use of the 4 COVID-19 vaccines approved in Canada as of March 25, 2021 (BNT162b2, mRNA-1273, ChAdOx1, and Ad26.COV2.S), over 4 virtual panel meetings.

Results. The CRA guideline panel suggests using COVID-19 vaccination in persons with ARD. The panel unanimously agreed that for the majority of patients, the potential health benefits of vaccination outweigh the potential harms in people with ARDs. The recommendation was graded as conditional because of low or very low certainty of the evidence on the effects in the population of interest, primarily due to indirectness and imprecise effect estimates. The panel felt strongly that persons with autoimmune rheumatic diseases who meet local eligibility should not be required to take additional steps compared to people without ARDs to obtain their vaccination. Guidance on medications, implementation, monitoring of vaccine uptake, and research priorities are also provided.

Conclusion. This recommendation will be updated over time as new evidence emerges, with the latest recommendation, evidence summaries, and $\mathrm{EtD}$ available on the CRA website.

Key Indexing Terms: autoimmune disease, clinical practice guideline, COVID-19, GRADE, rheumatic diseases, vaccination

${ }^{1}$ G.S. Hazlewood, MD, PhD, Associate Professor of Medicine, C. Barnabe, MD, MSc, Associate Professor of Medicine, C.E. Barber, MD, PhD, Assistant Professor of Medicine, Departments of Medicine and Community Health Sciences, Cumming School of Medicine, University of Calgary, Calgary, and Arthritis Research Canada, Richmond, British Columbia; ${ }^{2}$ J.P. Pardo, LDO, Managing Editor, Centre for Global Health, University of Ottawa, Ottawa, Ontario; ${ }^{3}$ O. Schieir, PhD, Canadian Early Arthritis Cohort Study, Toronto, Ontario; ${ }^{4}$ S. Bernatsky, MD, PhD, Professor of Medicine, Research Institute of the McGill University Health Centre (RI-MUHC), Montreal, Quebec; ${ }^{5}$ I. Colmegna, MD, Associate Professor of Medicine, Division of Rheumatology, Department of Medicine, McGill University, Montreal, Quebec; ${ }^{6}$ C. Hitchon, MD, MSc, Associate Professor of Medicine, Department of Internal Medicine, Max Rady College of Medicine, Rady Faculty of Health Sciences, University of Manitoba, Winnipeg, Manitoba; ${ }^{7}$ M. Loeb, $M D, M S c$, Professor, D. Mertz, MD, MSc, Associate Professor of Medicine, H.J. Schünemann, MD, MSc, PhD, Professor of Medicine and Clinical Epidemiology, Departments of Medicine and Health Research Methods, Evidence, and Impact, McMaster GRADE Centers, Hamilton; ${ }^{8}$ L. Proulx,
B.Com, D.P. Richards, PhD, Canadian Arthritis Patient Alliance, Toronto, Ontario; ${ }^{9} R$. Scuccimarri, MD, Associate Professor of Pediatrics, Division of Pediatric Rheumatology, Department of Pediatrics, McGill University, Montreal, Quebec; ${ }^{10} P$. Tugwell, MD, Professor of Medicine, A.L. Zhou, $M D$, Department of Medicine, University of Ottawa, Ottawa, Ontario; ${ }^{11}$ R.D. Mirza, MD, Department of Medicine, University of Toronto, Toronto, Ontario; ${ }^{12}$ R.P. Nikolic, BSc, Cumming School of Medicine, University of Calgary, Calgary, Alberta; ${ }^{13} M$. Thomas, BHSc, M. Ejaredar, PhD, Department of Community Health Sciences, Cumming School of Medicine, University of Calgary, Calgary, Alberta; ${ }^{14} \mathrm{H}$. Chase, University of Ottawa, Ottawa, Ontario; ${ }^{15}$ R. Nieuwlaat, MSc, PhD, Associate Professor, Department of Health Research Methods, Evidence, and Impact, McMaster University, Hamilton, Ontario, Canada.

$J P P$ has received funding from Canadian Rheumatology Association to Cochrane Musculoskeletal to provide methodological support for guideline development.

$C B$ has received honoraria for advisory boards (Gilead, Pfizer) and speaker fees (Sanofi, Novartis) in the past 3 years and was a nonvoting member 
Autoimmune rheumatic diseases (ARDs) include a range of chronic inflammatory conditions of the musculoskeletal and connective tissue systems, such as rheumatoid arthritis (RA), systemic lupus erythematosus (SLE), and vasculitis. Vaccines are an important part of the care of people living with ARDs. ${ }^{1,2}$ Owing to their disease, comorbidities associated with ARDs, and/or medications, people with ARDs may be at higher risk for infections or for developing worse outcomes from vaccine-preventable illnesses. ${ }^{3,4,5,6}$ Whereas live vaccines are not recommended for patients taking certain immune-suppressing medications owing to a potential risk of infection, inactivated vaccines may be safely administered, although their effectiveness may be diminished by these medications. ${ }^{1,2}$

Recently approved coronavirus 2019 (COVID-19) vaccines have brought tremendous promise to help end the pandemic that has caused an unprecedented impact on people and society. As of March 2021, 4 vaccines were approved for use in Canada, with more on the horizon. These include the mRNA vaccines BNT162b2 (Pfizer-BioNTech) and mRNA-1273 (Moderna), ${ }^{8}$ and the viral vector vaccines ChAdOx1 (AstraZeneca) ${ }^{9}$ and Ad26.COV2.S (Johnson \& Johnson). ${ }^{10}$ Through encapsulated lipid particles, mRNA vaccines deliver mRNA sequences for the spike protein of the SARS-CoV-2 virus. These mRNA sequences are translated into spike proteins in the recipient that elicit an immune response. ${ }^{7.8}$ Viral vector vaccines work similarly, but provide the genetic sequences for the spike protein on DNA, delivered through an attenuated adenovirus viral vector.

The clinical trials of the approved COVID-19 vaccines conducted to date largely excluded patients with autoimmune conditions and/or people taking immune-suppressing medications. ${ }^{78}$ Given the lack of direct evidence, recommendations on the vaccine in people with ARDs have varied. In Canada,

of the guidelines panel. $M L$ has received payment for advisory boards (Pfizer, Medicago, Sanofi, Seqirus) and data safety monitoring committee (CanSino Biologics), and was a nonvoting member of the guidelines panel. $L P$ serves as volunteer Vice President of Canadian Arthritis Patient Alliance which receives the majority of its funding from independent grants from pharmaceutical companies, and has received payment and travel support in the last 3 years from Lilly Canada (sharing patient perspectives at an event). DPR serves as volunteer Vice President of Canadian Arthritis Patient Alliance which receives the majority of its funding from independent grants from pharmaceutical companies. DPR's employer, Five02 Labs Inc., has received payment in the last 3 years from NovoNordisk (speaking about arthritis advocacy) and Lilly Canada (sharing patient story, participating in an advisory board). CH has received research funds from Research Manitoba, Health Sciences Centre Foundation, Pfizer, and UCB Canada for unrelated work, and was a nonvoting member of the guidelines panel. HJS serves as co-chair for the GRADE Working Group, has received funding from the Canadian Institutes of Health Research (CIHR; FRN VR4-172741), and was a nonvoting technical expert. The remaining authors report no conflicts of interest relevant to this article.

Address correspondence to Dr. G.S. Hazlewood, Departments of Medicine and Community Health Sciences, Cumming School of Medicine, University of Calgary, 3280 Hospital Drive NW, Calgary, AB T2N 4Z6, Canada.

Email: gshazlew@ucalgary.ca.

Accepted for publication May 14, 2021. the National Advisory Committee on Immunization (NACI) initially made a strong recommendation against the vaccine in people with autoimmune conditions owing to the lack of direct evidence, but this has since been modified to indicate that the vaccine may be offered if the benefits outweigh the risk for the individual patient. ${ }^{11}$ Other rheumatology groups, including the American College of Rheumatology (ACR), British Society for Rheumatology (BSR), and a position statement from the Canadian Rheumatology Association (CRA), have more actively encouraged vaccination for this population. ${ }^{12,13,14}$

Objective and need. The objective of these recommendations is to provide guidance for the use of COVID-19 vaccines in patients with ARDs. The guideline was approved by the Guidelines Committee of the CRA on January 15, 2021. The guideline was also deemed an urgent priority by the Canadian Arthritis Patient Alliance.

Target audience. The target audience is patients with ARDs, physicians, and other allied health professionals counseling patients regarding the COVID-19 vaccine.

Target population. This guideline is intended for individuals aged $\geq 16$ years with ARDs. ARDs are a diverse group of autoimmune conditions that commonly affect the joints and other organs or systems. This includes, but is not limited to, conditions such as RA, spondyloarthritis, SLE, myositis, polymyalgia rheumatica, Sjögren syndrome, and vasculitis (see Supplementary Text 1, available with the online version of this article, for additional, but not exhaustive, list of conditions). ${ }^{15}$ People with ARDs typically require long-term treatment with immune-modulating medications. ARDs do not include nonautoimmune conditions that can also affect joints or soft tissues, such as osteoarthritis or fibromyalgia.

This guideline is intended for people with ARDs, regardless of whether they are on current immune-modulating treatment or not. Treatments commonly used for these conditions include the following: glucocorticoids; synthetic disease-modifying antirheumatic drugs (DMARDs; e.g., methotrexate [MTX], leflunomide, sulfasalazine, hydroxychloroquine, chloroquine, and azathioprine); mycophenolic acid preparations; calcineurin inhibitors (cyclosporine, tacrolimus), the alkylating agent cyclophosphamide; biologic DMARDs (originator or biosimilars; infliximab, etanercept, adalimumab, certolizumab, golimumab, abatacept (ABA), tocilizumab, sarilumab, rituximab (RTX), secukinumab, ixekizumab, belimumab, anakinra, canakinumab); and targeted synthetic DMARDs (tofacitinib, baricitinib, upadacitinib).

Perspective. This guideline takes the perspective of the individual person living with an ARD. It does not consider population/health system issues related to vaccine prioritization or distribution.

\section{METHODS}

The CRA panel developed an initial recommendation for the approved mRNA vaccines between January 15, 2021, and February 13, 2021, and updated this to include the approved viral vector vaccines on March 25, 2021. This guideline was developed using the GRADE (Grading of Recommendations Assessment, Development, and Evaluation) approach, which provides a systematic process for appraising the certainty of evidence 
and grading the direction and strength of recommendations. ${ }^{16}$ Ethics approval was not required.

Organization and panel composition. The CRA assembled a guideline panel and included rheumatologists, methodologists, infectious disease physicians with expertise in vaccines, and 2 people living with ARDs (see Supplementary Table 1, available with the online version of this article). The panel included expertise in health equity, patient preferences, vaccination in patients with ARDs, vaccine hesitancy, lived experience with ARDs, evidence synthesis, and guideline development. Methodological support was provided by the Cochrane Musculoskeletal Centre for evidence synthesis and the McMaster GRADE Centre for guideline development. All panel meetings were held virtually by video calls. Seven panelists who did not have prior GRADE exposure or training completed a guideline development training course (International Guideline Development Credentialing \& Certification Program; inguide.org) with a focus on GRADE prior to the start of the guideline development process, which was offered free of charge.

Guideline funding and management of conflicts of interest. The guideline was supported by in-kind funding from the CRA, a nonprofit association that represents Canadian rheumatologists. The CRA also provides ongoing funding to the Cochrane Musculoskeletal Centre. Declarations of potential conflicts of interest (COI) were collected from all panelists and review team members (Supplementary Table 1, available with the online version of this article) using the International Committee of Medical Journal Editors form. The chair and co-chair, evidence review team, and all members of the voting panel were required to be free of any direct financial COI within the past 36 months, which meant no direct payments including research funding support from any manufacturers of COVID-19 vaccines that were currently approved or in development as of the date of the panel meetings. ${ }^{17}$ Expert panel members with COI were allowed to participate in the discussion but did not vote on any of the judgments (quality of evidence, evidence-to-decision [EtD] criteria, direction and strength of recommendation). The presence of direct financial COI was adjudicated by a staff member of the CRA separate from the guideline panel and was discussed with the chair and co-chair in the setting of ambiguity. The full list of submitted COIs are presented online (https://rheum.ca/resources/ cra-grade-recommendation-on-covid-19-vaccination-and-feedback-survey). Formulating clinical questions and determining outcomes of interest. The scope of the guideline was determined by the CRA to focus on whether COVID-19 vaccines should be used in persons with ARDs. No other questions were considered. Prior to the first meeting, a survey was circulated to the panel to agree on the definitions and specifications for population and interventions, and to rate the importance of the outcomes. In this initial guideline, the interventions were limited to approved COVID-19 vaccines as of March 25, 2021, but additional vaccines will be considered over time as they are approved for use in Canada.

Evidence review and grading of certainty of evidence. In order to identify the relevant data on COVID-19 vaccines, we used the resources available from the COVID-NMA initiative (https://covid-nma.com), ${ }^{18,19}$ a living evidence synthesis of all randomized controlled trials (RCTs) for COVID-19 vaccines. We supplemented our evidence review with indirect evidence of the efficacy and safety of other vaccines in people with ARDs (see Supplementary Text 2 for full details, available with the online version of this article). The certainty of the evidence for each outcome was categorized as very low, low, moderate, or high, according to GRADE methodology. ${ }^{16} \mathrm{RCTs}$ started as high quality and judgments were made whether to rate the certainty downward for the 5 GRADE domains: risk of bias, inconsistency, indirectness, publication bias, and imprecision..$^{20}$ Observational evidence started as low quality and could be rated downward for the same 5 domains, or could be rated upwards for 3 additional domains: presence of large effects, dose-response relationship, and the effect of plausible residual confounding. ${ }^{20}$

Development of recommendation. A recommendation for the 2 mRNA vaccines (BNT 162b2 and mRNA-1273) was first developed over 3 virtual panel meetings and published online. Subsequently, a recommendation for the Ad26.COV2.S and ChAdOx1 vaccines was developed during an additional panel meeting and the guideline was updated. GRADE EtD profiles were developed in GRADEpro software (https://gradepro.org). The EtDs included the summary of the evidence for desirable and undesirable effects with overall certainty of the evidence rating, and additional EtD domains of patient preferences and values, resource utilization, equity, acceptability, and feasibility. ${ }^{21}$ Differences between the vaccines were highlighted. The EtDs were prepared by a central team (GSH, JP, CB, RN) and reviewed by panel members prior to meetings. During the panel meetings, the panel discussed each $\mathrm{EtD}$ domain and then voted privately to the panel co-chair for each required judgment. The panel discussed the votes, and reached a consensus judgment, which required a simple majority ( $>50 \%$ ) of the votes if there was disagreement. Following the EtD judgments, they then voted on the direction and strength of the recommendation. A simple majority (> 50\%) was required to determine the direction of the recommendation, and development of a strong recommendation required $\geq 80 \%$ of the panel to agree.

How to read this guideline. In the GRADE approach, recommendations are categorized as "strong" or "conditional."22 A strong recommendation means that all or almost all persons would choose that intervention. A conditional recommendation means that the majority of individuals in this situation would want the suggested course of action, but many would not (Table 1).

Living guideline. This guideline will be updated in a "living" fashion over time. Modifications will be planned when new vaccines are approved in Canada, or when new or higher-certainty evidence (e.g., on an outcome in the population of interest) emerges. To identify new evidence, we will leverage an existing effort to identify and map national and international vaccine recommendations, ${ }^{23}$ living evidence reviews of COVID-19 vaccine clinical trials, ${ }^{18,19}$ and a planned Cochrane review of COVID-19 vaccine safety and efficacy in patients with ARDs.

Public commenting. The draft guideline was published for public commenting on the CRA website on February 13, 2021: https://rheum.ca/ resources/cra-grade-recommendation-on-covid-19-vaccination-and-feedback-survey. The public comments will be reviewed on an ongoing basis and considered in future updates.

How to use this guideline. This recommendation is intended to help clinicians and patients make decisions regarding COVID-19 vaccination. It is not meant to replace clinical judgment. The recommendation should always be presented with the accompanying remarks to aid in interpretation. Guideline users should be aware that the recommendation is subject to change over time in a living fashion as new evidence emerges and should always consult the CRA website (https://rheum.ca/resources/ cra-grade-recommendation-on-covid-19-vaccination-and-feedback-survey) for the latest version.

\section{RESULTS}

Should COVID-19 vaccination vs no COVID-19 vaccination be used for persons with ARD?

Recommendation. The CRA guideline panel suggests using COVID-19 vaccination in persons with ARDs (conditional recommendation; low certainty of the evidence about effects of BNT 162b2 [Pfizer-BioNTech], mRNA-1273 [Moderna], and Ad26.COV2.S [Johnson \& Johnson]; very low certainty for ChAdOx1 [AstraZeneca]).

Remark. This recommendation is based on evidence for the approved COVID-19 vaccines BNT 162 b2 (Pfizer-BioNTech), mRNA-1273 (Moderna), Ad26.COV2.S (Johnson \& Johnson), and ChAdOx1 (AstraZeneca). The recommendation needs to be viewed in the context of any restrictions to vaccine use for 
Table 1. Interpretation of strong and conditional recommendations.

\begin{tabular}{|c|c|c|}
\hline Implications for: & Strong Recommendation & Conditional Recommendation \\
\hline Patients & $\begin{array}{l}\text { Most individuals in this situation would want the } \\
\text { recommended course of action, and only a small } \\
\text { proportion would not. }\end{array}$ & $\begin{array}{l}\text { The majority of individuals in this situation would want the suggested } \\
\text { course of action, but many would not. Decision aids may be useful in } \\
\text { helping patients to make decisions consistent with their individual risks, } \\
\text { values, and preferences. }\end{array}$ \\
\hline Clinicians & $\begin{array}{l}\text { Most individuals should follow the recommended } \\
\text { course of action. Formal decision aids are not likely } \\
\text { to be needed to help individual patients make decisions } \\
\text { consistent with their values and preferences. }\end{array}$ & $\begin{array}{l}\text { Recognize that different choices will be appropriate for individual } \\
\text { patients and that you must help each patient arrive at a management } \\
\text { decision consistent with his or her values and preferences. Decision } \\
\text { aids may be useful in helping individuals to make decisions consistent } \\
\text { with their individual risks, values, and preferences. }\end{array}$ \\
\hline Policy makers & $\begin{array}{l}\text { The recommendation can be adopted as policy in } \\
\text { most situations. Adherence to this recommendation } \\
\text { according to the guideline could be used as a quality } \\
\text { criterion or performance indicator. }\end{array}$ & $\begin{array}{l}\text { Policymaking will require substantial debate and involvement of various } \\
\text { stakeholders. Performance measures should assess if decision making is } \\
\text { appropriate. }\end{array}$ \\
\hline
\end{tabular}

that alters the recommendations.

the general public set by national or provincial bodies that may change over time.

Primary justification. The panel was unanimous that for the majority of patients, the potential benefits outweigh the potential harms in people with ARDs. The recommendation was graded as conditional because of uncertainty about the effects in the population of interest.

Primary implementation consideration for policy makers and providers. Persons with ARDs who meet local eligibility criteria for COVID-19 vaccination should not be denied access to vaccination and should not be required to take additional steps to obtain their vaccination compared to people without ARDs.

\section{Summary of the evidence}

Benefits. The benefits of the COVID-19 vaccine were considered large for preventing symptomatic COVID-19 (all vaccines) and severe or critical COVID-19 (BNT 162b2, mRNA-1273, Ad26. COV2.S; Tables 2-4). The panel unanimously agreed that there was an overall large magnitude of benefits for all vaccines. Some people with ARDs may have less protection from the vaccine, based on the medications they are taking. From data of other (non-COVID-19) vaccines, MTX, mycophenolate mofetil, tofacitinib, and prednisone ( $\geq 10 \mathrm{mg} /$ day) have also been shown to attenuate vaccine-induced responses. ${ }^{2}$ A single small study with $\mathrm{ABA}$ and influenza vaccine also showed decreased immunogenicity. ${ }^{7}$ Emerging data in COVID-19 vaccines suggest serological responses may also be reduced in patients taking certain medications $s^{24,25}$; however, given the large magnitude of benefit, it is likely that the benefits of the vaccine will still be large for most patients with ARD. One potential exception is with patients on RTX treatment, as notable decreases in immunogenicity have been seen post-influenza vaccine $e^{2,8,9,10,11}$ (see subgroup considerations below).
The panel also discussed how the benefits of COVID-19 vaccination in absolute terms will vary based on an individual's risk of acquiring COVID-19, which will depend on place of residence, community transmission of COVID-19, occupation, and social and family contacts. The benefits of preventing severe disease will also vary by a patient's individual risk factors for COVID-19. While data have not suggested ARDs are an independent risk factor for severe COVID-19, ${ }^{12,13,14,15,16}$ many persons with ARDs are older and have higher rates of comorbidities, ${ }^{17,18,19}$ which are associated with more severe disease from COVID-19. In people with ARDs, higher disease activity and certain medications (including prednisone $\geq 10 \mathrm{mg} /$ day) have been associated with an increased risk of hospitalization and/ or death in those with COVID-19 infection. ${ }^{20,21}$ Finally, ARDs are also more prevalent in populations at risk for inequities in COVID-19 infection rates and outcomes (e.g., Indigenous populations). ${ }^{22}$

Additional potential benefits of the vaccine include avoiding isolation requirements associated with contracting COVID-19 infection, protecting loved ones, improved herd immunity, and helping end the pandemic. It was noted though that even if people receive the vaccine, it would still be important to continue following public health recommendations and not to assume they are protected from COVID-19. While this guideline took the perspective of the individual patient decision, additional societal benefits could include increased herd immunity, reduction of virus persistence and mutations, and reduction of infection.

Harms. The point estimate for serious adverse events (AEs) was either of negligible magnitude (mRNA vaccines) or favored the vaccine (Ad26.COV2.S and ChAdOx1; Tables 2-4). The point estimate for autoimmune AEs favored the mRNA vaccines (Table 2) and was not well reported for the other vaccines 
Table 2. Summary of findings table for BNT $162 \mathrm{~b} 2$ and mRNA-1273 (mRNA) COVID-19 vaccines in people with autoimmune rheumatic diseases. ${ }^{\dagger}$

\begin{tabular}{|c|c|c|c|c|c|}
\hline \multirow[t]{3}{*}{ Outcomes } & \multicolumn{2}{|c|}{ Anticipated Absolute Effects* (95\% CI) } & \multirow{3}{*}{$\begin{array}{c}\text { Relative Effect } \\
\quad(95 \% \mathrm{CI})\end{array}$} & \multirow{3}{*}{$\begin{array}{c}\text { No. of Participants } \\
\text { (Studies) }\end{array}$} & \multirow{3}{*}{$\begin{array}{c}\text { Certainty of the Evidence, } \\
\text { GRADE }\end{array}$} \\
\hline & Risk With & Risk With & & & \\
\hline & Placebo & COVID-19 Vaccine & & & \\
\hline Severe or critical disease & 110 per 100,000 & $\begin{array}{c}4 \text { per } 100,000 \\
(1-21)\end{array}$ & $\begin{array}{c}\text { RR } 0.04 \\
(0.01-0.19)\end{array}$ & $\begin{array}{c}70,780 \\
(2 \mathrm{RCTs})\end{array}$ & $\begin{array}{l}\bigoplus \bigoplus \bigoplus O \\
\text { MODERATE }\end{array}$ \\
\hline Severe adverse events & 717 per 100,000 & $\begin{array}{c}739 \text { per } 100,000 \\
(624-875)\end{array}$ & $\begin{array}{c}\text { RR 1.03 } \\
(0.87-1.22)\end{array}$ & $\begin{array}{c}73,603 \\
(2 \mathrm{RCTs})\end{array}$ & $\begin{array}{c}\bigoplus \bigoplus{ }_{\mathrm{LOW}}^{\mathrm{a}, \mathrm{b}} \\
\end{array}$ \\
\hline $\begin{array}{l}\text { Autoimmune adverse } \\
\text { events }\end{array}$ & 13 per 100,000 & $\begin{array}{c}7 \text { per } 100,000 \\
(1-73)\end{array}$ & $\begin{array}{c}\text { RR } 0.50 \\
(0.05-5.51)\end{array}$ & $\begin{array}{c}30,351 \\
(1 \mathrm{RCT})\end{array}$ & $\underbrace{\bigoplus \text { a,b }}_{\text {VERY LOW }}$ \\
\hline $\begin{array}{l}\text { Exacerbation of } \\
\text { preexisting disease }\end{array}$ & $\begin{array}{l}\text { Immunization did } \mathrm{r} \\
\text { significant worsenir } \\
\text { metaanalysis evalua } \\
\text { pneumococcal vacc } \\
\text { demonstrated that i } \\
\text { effect on the SLED }\end{array}$ & $\begin{array}{l}\text { nerally cause clinically } \\
\text { anderlying ARDs. A } \\
\text { ne effect of influenza and } \\
n \text { in systemic SLE } \\
\text { nization had no significant } \\
\text { re. }\end{array}$ & & $\begin{array}{c}759 \\
\text { (20 observational studies) }\end{array}$ & $\underset{\text { VERY LOW }}{\bigoplus O O O}$ \\
\hline
\end{tabular}

GRADE Working Group grades of evidence: (1) high certainty: we are very confident that the true effect lies close to that of the estimate of the effect; (2) moderate certainty: we are moderately confident in the effect estimate (the true effect is likely to be close to the estimate of the effect, but there is a possibility that it is substantially different); (3) low certainty: our confidence in the effect estimate is limited (the true effect may be substantially different from the estimate of the effect); (4) very low certainty: we have very little confidence in the effect estimate (the true effect is likely to be substantially different from the estimate of effect). ${ }^{\dagger}$ Interactive table available online at https://gdt.gradepro.org/presentations/\#/isof/isof_d4a3bbe1-0a4f-42c8-93b3-8b71ce0a64f9-1620580039869?_ $\mathrm{k}=\mathrm{z} 1 \mathrm{jtm} 0 .{ }^{*}$ The risk in the intervention group (and its $95 \% \mathrm{CI}$ ) is based on the assumed risk in the comparison group and the relative effect of the intervention (and its 95\% CI). ${ }^{a}$ Downgraded 1 level for indirectness due to the population of interest being excluded from the trials. ${ }^{b}$ Downgraded 1 level for imprecision due to CI including serious benefits and serious harms. ${ }^{\mathrm{c}}$ Downgraded 1 level for inconsistency due to extreme heterogeneity $(\mathrm{chi}$-square $=513.92 ; \mathrm{df}=1$ $[P<0.00001]) ; \mathrm{I}^{2}=100 \% .{ }^{\mathrm{d}}$ Downgraded 1 level for indirectness due to vaccine of interest not included in the studies. ARD: autoimmune rheumatic disease; COVID-19: coronavirus disease 2019; df: degrees of freedom; GRADE: Grading of Recommendations Assessment, Development, and Evaluation; RR: risk ratio; RCT: randomized controlled trial; SLE: systemic lupus erythematosus; SLEDAI: SLE Disease Activity Index.

(Table 3, Table 4). The increase in any AEs seen with the mRNA and Ad26.COV2.S vaccines was judged to be of trivial magnitude, given that they were largely expected injection site or systemic reactions to the vaccine. The decrease in any AEs seen with the ChAdOx1 vaccine was judged to be of trivial magnitude but the AE data for the ChAdOx1 vaccine was difficult to interpret because of the mixed comparator (meningococcal vaccine or placebo). There was no evidence from studies in other vaccines that immunization results in a significant increase in disease activity (flares) in patients with ARDs, although the available data were limited and heterogeneous in terms of types of vaccines, flare definitions, and populations studied (see Supplementary Figure 1 and Supplementary Tables 2-4, available with the online version of this article). ${ }^{2,23}$ The panel also discussed (March 25, 2021) the recent reports of very rare occurrences of vaccine-induced thrombotic thrombocytopenia identified with the ChAdOx1 vaccine $e^{26,27,28}$ and, subsequently, with the Ad26.COV2.S vaccine. ${ }^{29}$ The panel felt that guidance from national/provincial bodies regarding the use of vaccines in the setting of new safety data should be followed; safety issues identified in the general population would also apply to people with ARDs.

Certainty of evidence. The certainty of evidence for each outcome is presented in the evidence profiles (Tables 2-4). All outcomes were rated down 1 level for indirectness given that patients with autoimmune disease and people on immunosuppressants were largely excluded from COVID-19 vaccine clinical trials. ${ }^{7.8}$ There was moderate quality evidence for benefits and low (mRNA, Ad26.COV2.S) or very low (ChAdOx1) quality evidence for harms, which resulted in assigning an overall low or very low quality of evidence, primarily due to additional concerns about imprecision. 
Table 3. Summary of findings table for Ad26.COV2.S COVID-19 vaccine in people with autoimmune rheumatic diseases.

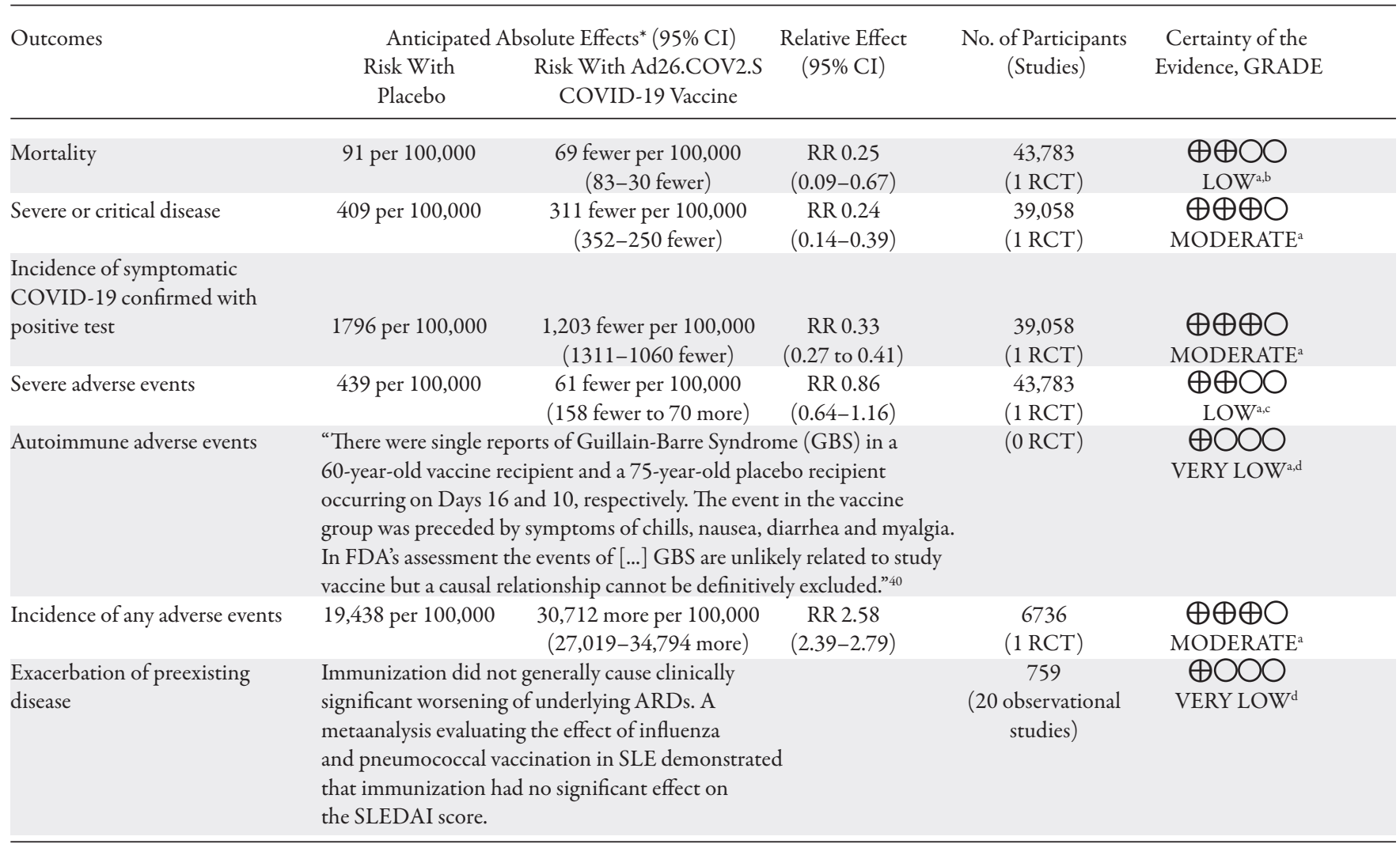

GRADE Working Group grades of evidence: (1) high certainty: we are very confident that the true effect lies close to that of the estimate of the effect; (2) moderate certainty: we are moderately confident in the effect estimate (the true effect is likely to be close to the estimate of the effect, but there is a possibility that it is substantially different); (3) low certainty: our confidence in the effect estimate is limited (the true effect may be substantially different from the estimate of the effect); (4) very low certainty: we have very little confidence in the effect estimate (the true effect is likely to be substantially different from the estimate of effect). ${ }^{\dagger}$ Interactive table available online at https://gdt.gradepro.org/presentations/\#/isof/isof_ee6e5a9d-f819-4903-b704-3df58e02dfa7-1620580142011 ? $\mathrm{k}=\mathrm{t} 82 \mathrm{vzx} .{ }^{*}$ The risk in the intervention group (and its $95 \% \mathrm{CI}$ ) is based on the assumed risk in the comparison group and the relative effect of the intervention (and its 95\% CI). ${ }^{a}$ Downgraded 1 level for indirectness as autoimmune patients were not included in the trials. ${ }^{b}$ Downgraded 1 level for imprecision due to small number of events. ${ }^{c}$ Downgraded 1 level for imprecision due to CI including serious benefits and serious harms. ${ }^{\mathrm{d}}$ Imprecision downgraded by 2 levels due to scarcity of data and insufficient reporting. ARD: autoimmune rheumatic disease; COVID-19: coronavirus disease 2019; GRADE: Grading of Recommendations Assessment, Development, and Evaluation; RR: risk ratio; RCT: randomized controlled trial; SLE: systemic lupus erythematosus; SLEDAI: SLE Disease Activity Index.

Other EtD criteria and considerations. The full EtD frameworks are available online through GRADEpro:

- BNT $162 \mathrm{~b} 2$ and mRNA-1273 (mRNA): https://guidelines.gradepro.org/profile/QnfX_seQRDw

- Ad26.COV2.S: https://guidelines.gradepro.org/profile/ $\mathrm{FH} 3 \mathrm{el} 5 \mathrm{YsCn}$

- ChAdOx1: https://guidelines.gradepro.org/profile/ Wq9afPW638Q

The panel judged that a recommendation for the vaccine would be expected to increase health equity, as ARDs and COVID-19 are more prevalent and can be more severe in populations at risk for inequities, for whom COVID-19 vaccines are being prioritized..$^{30,31,32}$ Vaccination may also increase health equity by ensuring people with ARDs are able to reengage with society at a similar rate to people without $\mathrm{ARDs}$ as the pandemic eases (i.e., not be "left behind"). This may help lessen challenges that people with ARDs already face with work, family, and social life. The panel also discussed that in Canada, some patients with ARDs have had difficulty accessing the COVID-19 vaccine, despite being eligible based on provincial vaccine priority groups. The Ad26.COV2.S vaccine was judged to further increase health equity, as the single dose will be easier to administer. The panel felt strongly that people with ARDs should be able to access the COVID-19 vaccine without any additional barriers. People should be informed about the lack of direct evidence but should not be required to take additional steps to obtain their vaccination (for instance, requiring a physician letter or documentation). Vaccine clinics should be accessible to persons with disabilities given the functional and mobility impairments of people living with ARDs. Creating additional requirements was judged to increase inequities.

Conclusion. The panel balanced the moderate certainty in the large vaccine benefits against the low/very low certainty of evidence for harm. Although the magnitude of the best estimate 
Table 4. Summary of findings table for ChAdOx1 COVID-19 vaccine in people with autoimmune rheumatic diseases. ${ }^{\dagger}$

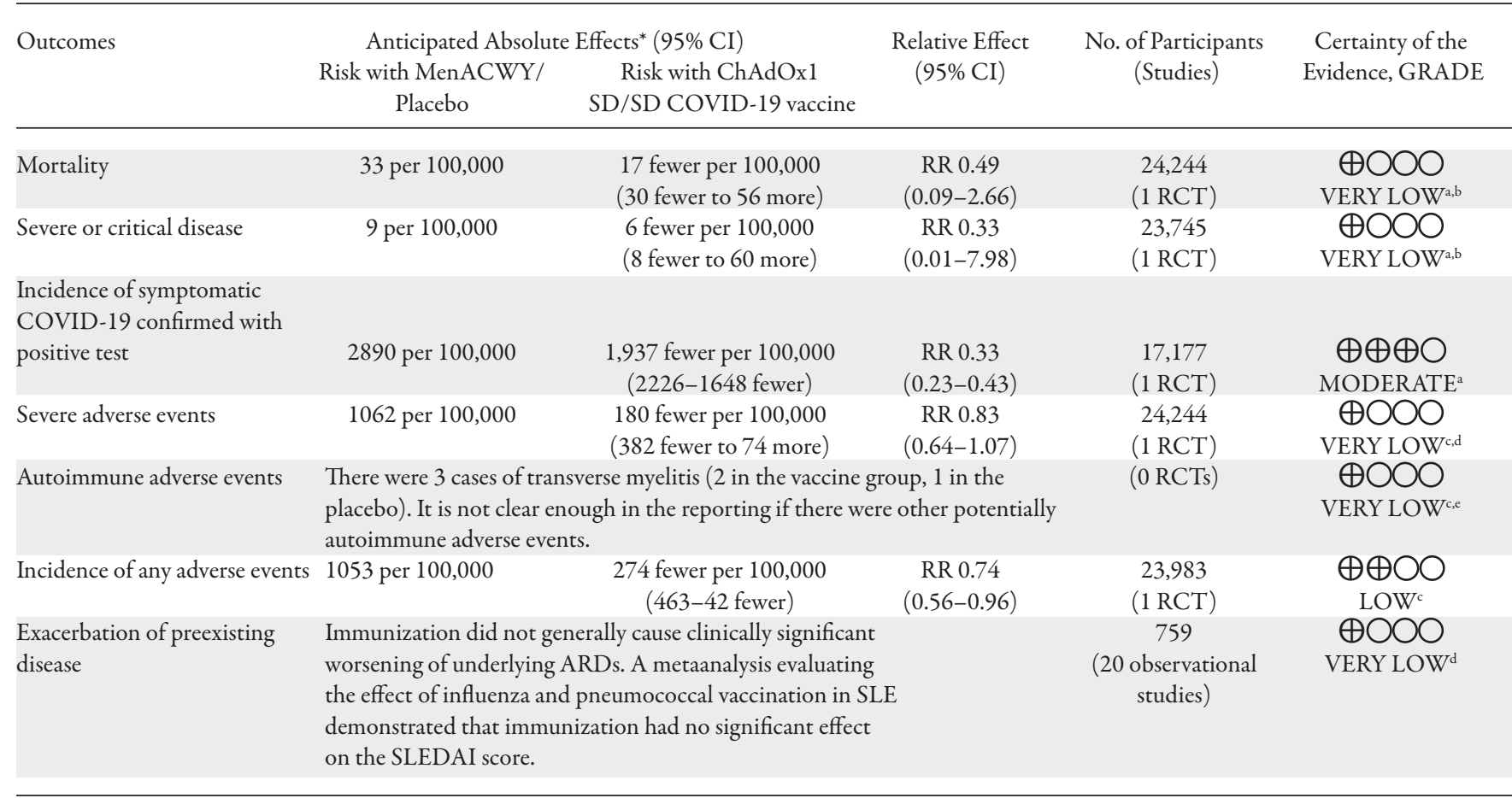

GRADE Working Group grades of evidence: (1) high certainty: we are very confident that the true effect lies close to that of the estimate of the effect; (2) moderate certainty: we are moderately confident in the effect estimate (the true effect is likely to be close to the estimate of the effect, but there is a possibility that it is substantially different); (3) low certainty: our confidence in the effect estimate is limited (the true effect may be substantially different from the estimate of the effect); (4) very low certainty: we have very little confidence in the effect estimate (the true effect is likely to be substantially different from the estimate of effect). ${ }^{\dagger}$ Interactive table available online at https://gdt.gradepro.org/presentations/\#/isof/isof_94981a15-9190-45fb-8198-75c95cbd5328-16205801073444? $\mathrm{k}=$ dlzkfe. ${ }^{*}$ The risk in the intervention group (and its $95 \% \mathrm{CI}$ ) is based on the assumed risk in the comparison group and the relative effect of the intervention (and its 95\% CI). a Downgraded 1 level because autoimmune patients were excluded from the trials. ${ }^{\text {b }}$ Imprecision downgraded by 2 levels due to wide CI consistent with the possibility for benefit and the possibility for harm and few events. ${ }^{c}$ Downgraded 2 levels for indirectness because autoimmune patients were excluded from the trials and the control groups mixed a placebo and an active component (meningitis vaccine). ${ }^{\mathrm{d}}$ Imprecision downgraded by 1 level due to wide CI consistent with the possibility for no effect and the possibility for benefit. ${ }^{e}$ Imprecision downgraded by 2 levels due to scarcity of data and insufficient reporting. ARD: autoimmune rheumatic disease; COVID-19: coronavirus disease 2019; GRADE: Grading of Recommendations Assessment, Development, and Evaluation; MenACWY: meningococcal vaccine; RR: risk ratio; RCT: randomized controlled trial; SD/SD: standard dose; SLE: systemic lupus erythematosus; SLEDAI: SLE Disease Activity Index.

of harms was judged to be trivial, the uncertainty in the evidence led to a conditional recommendation.

\section{Subgroup considerations/medications}

People taking RTX. Based on serological studies from other vaccines, RTX is expected to decrease immunogenicity. ${ }^{2}$ Prior guidelines for other vaccines in patients with ARDs have recommended that immunization be deferred to $\geq 4-5$ months after the last dose and at least 4 weeks prior to the subsequent dose of RTX. ${ }^{2}$

People taking other DMARDs. Some other DMARDs may reduce protection from the vaccine. Given the large magnitude of benefit of the COVID-19 vaccines, it is likely that the benefits of the vaccine will still be large for most patients with ARDs. Continuing medications will often be the safest option to prevent disease flares until more evidence is available. This is in line with guidance from the BSR..$^{14}$ Recent guidance from the ACR recommended holding some medications (MTX, Janus kinase inhibitors, $\mathrm{ABA}$ ) around the time of COVID-19 vaccination, but the full guideline had not been published and the evidence supporting this was unclear. ${ }^{12}$ The CRA COVID-19 guideline panel did not feel that this guidance could be endorsed at this point but will review new evidence as it emerges. Any decision to hold medications should be discussed between a patient and their rheumatologist or healthcare team.

Pregnant and breastfeeding women. Additional considerations apply for pregnant and breastfeeding women, and should be discussed between a patient and their perinatal care team. These were not covered in the scope of this guideline.

\section{Implementation considerations}

As vaccine access is determined by provincial health authorities, it will be essential to ensure people with ARDs do not face unnecessary additional barriers to vaccine access. For instance, people with ARDs should not be required to obtain a physician letter as proof of an informed decision discussion. A decision tool, co-developed by the CRA and the Canadian Arthritis Patient Alliance to support decision making for the COVID-19 
vaccine in people with $A R D s$ is available at https://rheum.ca/ decision-aid. ${ }^{13}$ People with ARDs may also have mobility limitations and appropriate access to vaccine clinics should be ensured. Finally, the available data are for on-label dosing (doses separated by 1 month for mRNA and ChAdOx1 vaccines). Given that people with ARDs may have reduced vaccine-induced immunity, the benefits of off-label dosing may be lower compared to people without ARDs. As such, the CRA has recently advocated for on-label dosing for immunosuppressed patients. ${ }^{13}$

\section{Monitoring and evaluation}

Monitoring of vaccine uptake should occur in people with ARDs, including populations at risk of inequity. Low uptake may point to barriers to access or hesitancy. The frequency of serious AEs, disease flares, and COVID-19 infection/serious outcomes should be followed in patients with ARDs who do and do not receive the vaccine. People with ARDs should be encouraged to track their immunization history using an online Canadian vaccination tracker, developed with funding support from the Public Health Agency of Canada (www.canimmunize.ca).

Research recommendations. The panel proposed several research priorities, summarized in Table 5.

\section{DISCUSSION}

In this paper we present the CRA's recommendation for COVID-19 vaccination in people with ARDs. This recommendation will be updated in a living fashion over time as new evidence and new vaccines are approved.

A strength of our approach is the use of GRADE methodology. We present our full EtD framework, which provides transparency to our process and outlines the rationale supporting our recommendation. We included appropriate stakeholders throughout the process, including rheumatologists, experts in vaccination, methodologists, and people with lived experience of ARDs. We identified research priorities along with a rationale on how the research links to key aspects of our EtD process and how future evidence might affect the recommendation. Our evidence review included the pivotal trials of the COVID-19 vaccines, as well as other indirect evidence on vaccine safety and efficacy in patients with ARDs. Limitations of our approach include the use of secondary reviews, which may have resulted in missing some newer studies of other non-COVID-19 vaccines, although this would not have changed our recommendation, as the data would still be indirect. We focused on currently approved vaccines, but others will be added as they are approved in Canada.

Our recommendation in support of COVID-19 vaccination aligns with those of other rheumatology groups. The BSR recommended the use of the COVID-19 vaccine for immunosuppressed patients, although no evidence review or $\mathrm{EtD}$ process was presented. ${ }^{14}$ The ACR developed COVID-19 vaccination guidance through a Delphi process and evidence review. ${ }^{12}$ The ACR recommended that patients should receive COVID-19 vaccination, also making a conditional recommendation. As the groups used different approaches, it is difficult to directly compare language. A conditional recommendation should not be interpreted as less supportive of vaccination, particularly in patients at higher risk of COVID-19 infection outcomes, which include many patients with ARDs. In these patients, the benefits of COVID-19 vaccination clearly outweigh any theoretical risks. Our conditional recommendation reflects the lack of direct evidence and as such, some patients at particularly low risk of severe COVID-19 outcome may prefer to wait until additional direct evidence is available. We have developed an information sheet to support decision making for patients with ARDs. ${ }^{13}$ This is freely available and can be modified and adapted on request to other populations.

Important differences are present between our approach and the approach from NACI in Canada, despite both using GRADE. ${ }^{11}$ Most notably, NACI cited an absence of evidence in patients with autoimmune conditions when making a recommendation initially against the vaccine. However, a lack of direct evidence should not be interpreted as a complete absence of evidence. GRADE provides guidance in this regard. In situations where the population of interest does not match the population studied in the clinical trials, reviewers need to decide whether to rate the certainty of evidence down for indirectness. ${ }^{33}$ This

Table 5. Research priorities related to COVID-19 vaccines in people with autoimmune rheumatic diseases.

Research Priority

Observational evidence on the frequency of harms (in particular serious AEs/serious disease flares) in people with ARDs

Evidence comparing the frequency of serious AEs and autoimmune AEs in people with ARDs to those without ARDs

Evidence on the benefits (both clinical outcomes and serological studies) in people with ARDs on different medications, including the impact of off-label dosing on effectiveness

Evidence on patient values preferences for the benefits and harms across different patient populations

Understanding vaccine hesitancy and barriers to vaccine access faced by persons with ARDs

Understanding vaccine benefits and harms in populations at risk for inequities
Rationale

If very infrequent, may lower the importance of these outcomes

If not different with sufficient certainty, the panel may decide not to rate the quality of evidence for harms down for indirectness

May help inform decisions regarding whether to hold medications around the time of vaccination and recommendations on optimal dosing intervals for 2-dose vaccines

Will help inform the relative importance of the outcomes

Will help inform strategies to address vaccine hesitancy

Will help inform strategies to address inequity in vaccine access and uptake

AE: adverse event; ARD: autoimmune rheumatic disease; COVID-19: coronavirus disease 2019. 
situation is not uncommon in clinical medicine. Indeed, in rheumatology practice, patients treated in practice often differ from those included in clinical trials. ${ }^{34,35}$ GRADE states that "one should not rate down for population differences unless one has compelling reason to think that the biology in the population of interest is so different from that of the population tested that the magnitude of effect will differ substantially." ${ }^{33}$ Researchers should also be cautious not to rate the quality of evidence down for indirectness without sufficient rationale, so as not to increase inequities in vulnerable populations who are often excluded from clinical trials. ${ }^{36}$ Our panel decided to rate the certainty of evidence down 1 level for indirectness for all outcomes. The fact that these vaccines employed a new technology influenced this, but the safety of other vaccines in people with ARDs and very low rates of autoimmune AEs seen in the clinical trials of COVID-19 vaccines (with no differences between groups) tempered this.

One of the key considerations with the vaccine is in regard to health equity. The COVID-19 pandemic has disproportionately affected many vulnerable groups including people living with ARDs. ${ }^{37}$ This community has faced drug shortages, disruptions in accessing healthcare professionals, and medication supply restrictions, as well as increased anxiety and fear of what contracting COVID-19 will mean to them and their families. ${ }^{38,39}$ The remarkable development of highly effective COVID-19 vaccines has provided hope to patients with ARDs. The panel felt very strongly and unanimously that all patients with ARDs should not have any additional barriers to vaccine access, such as requiring a physician's letter, which may be difficult for some vulnerable populations, especially given some difficulties in accessing healthcare practitioners. As our recommendation focused on the individual patient decision, rather than a population perspective, we did not consider issues of vaccine prioritization. We do note that other groups have recommended patients with ARDs are in a higher priority group, ${ }^{12}$ and this has recently been implemented in several provinces in Canada. We are certainly supportive of this decision and believe it will help lessen the impact on vulnerable communities.

In summary, we present the CRA's recommendation for the use of COVID-19 vaccines in patients with ARDs. We provide a recommendation for the use of COVID-19 vaccines, as well as subgroup considerations for patients taking certain medications. This recommendation will be updated over time, with the latest recommendation hosted on the CRA website.

\section{ACKNOWLEDGMENT}

The authors thank Sue Ranta and the CRA for administrative support during the development and dissemination of the guideline, Alexa Au for assistance with the evidence review, and Walter Maksymowych for his thoughtful review of the manuscript. We thank Evidence Prime for providing a complimentary license for GRADEpro software.

\section{ONLINE SUPPLEMENT}

Supplementary material accompanies the online version of this article.

\section{REFERENCES}

1. Furer V, Rondaan C, Heijstek MW, Agmon-Levin N, van Assen
S, Bijl M, et al. 2019 update of EULAR recommendations for vaccination in adult patients with autoimmune inflammatory rheumatic diseases. Ann Rheum Dis 2020;79:39-52.

2. Papp KA, Haraoui B, Kumar D, Marshall JK, Bissonnette R, Bitton A, et al. Vaccination guidelines for patients with immune-mediated disorders on immunosuppressive therapies. J Cutan Med Surg 2019;23:50-74.

3. Yun H, Yang S, Chen L, Xie F, Winthrop K, Baddley JW, et al. Risk of herpes zoster in autoimmune and inflammatory diseases: implications for vaccination. Arthritis Rheumatol 2016; 68:2328-37.

4. Shigayeva A, Rudnick W, Green K, Chen DK, Demczuk W, Gold WL, et al. Invasive pneumococcal disease among immunocompromised persons: implications for vaccination programs. Clin Infect Dis 2016;62:139-47.

5. Doran MF, Crowson CS, Pond GR, O'Fallon WM, Gabriel SE. Frequency of infection in patients with rheumatoid arthritis compared with controls: a population-based study. Arthritis Rheum 2002;46:2287-93.

6. Chang CC, Chang YS, Chen WS, Chen YH, Chen JH. Effects of annual influenza vaccination on morbidity and mortality in patients with systemic lupus erythematosus: a nationwide cohort study. Sci Rep 2016;6:37817.

7. Polack FP, Thomas SJ, Kitchin N, Absalon J, Gurtman A, Lockhart S, et al; C4591001 Clinical Trial Group. Safety and efficacy of the BNT162b2 mRNA COVID-19 vaccine. N Engl J Med 2020;383:2603-15.

8. Baden LR, El Sahly HM, Essink B, Kotloff K, Frey S, Novak R, et al; COVE Study Group. Efficacy and safety of the mRNA-1273 SARS-CoV-2 vaccine. N Engl J Med 2021;384:403-16.

9. Voysey M, Clemens SAC, Madhi SA, Weckx LY, Folegatti PM, Aley PK, et al; Oxford COVID Vaccine Trial Group. Safety and efficacy of the ChAdOx $1 \mathrm{nCoV}-19$ vaccine (AZD1222) against SARS-CoV-2: an interim analysis of four randomised controlled trials in Brazil, South Africa, and the UK. Lancet 2021;397:99-111.

10. Sadoff J, Gray G, Vandebosch A, Cardenas V, Shukarev G, Grinsztejn B, et al; ENSEMBLE Study Group. Safety and efficacy of single-dose Ad26.COV2.S vaccine against COVID-19. N Engl J Med 2021 Apr 21 (E-pub ahead of print).

11. National Advisory Committee on Immunization (NACI). Recommendations on the use of COVID-19 vaccines. December 23, 2020 [Internet. Accessed May 17, 2021]. Available from: www. canada.ca/en/public-health/services/immunization/ national-advisory-committee-on-immunization-naci/ recommendations-use-covid-19-vaccines.html

12. American College of Rheumatology (ACR) COVID-19 Vaccine Clinical Guidance Task Force. COVID-19 vaccine clinical guidance summary for patients with rheumatic and musculoskeletal diseases. [Internet. Accessed May 17, 2021]. Available from: www. rheumatology.org/Portals/0/Files/COVID-19-Vaccine-ClinicalGuidance-Rheumatic-Diseases-Summary.pdf

13. Canadian Rheumatology Association. Canadian Rheumatology Association position statement on Covid-19 vaccination. [Internet. Accessed May 17, 2021]. Available from: mcusercontent. com/912adf891f7fdc4dfefb739ba/files/0df3fde1-a4a3-4f24-b6f5eb4b3e053c11/CRA_Position_Statement_on_COVID_19_ Vaccination_v2_FINAL.pdf

14. British Society for Rheumatology. COVID-19 guidance. [Internet. Accessed May 17, 2021]. Available from: www.rheumatology.org. uk/practice-quality/covid-19-guidance

15. Goldblatt F, O'Neill SG. Clinical aspects of autoimmune rheumatic diseases. Lancet 2013;382:797-808.

16. Guyatt G, Oxman AD, Akl EA, Kunz R, Vist G, Brozek J, et al. 
GRADE guidelines: 1. Introduction-GRADE evidence profiles and summary of findings tables. J Clin Epidemiol 2011;64:383-94.

17. Craven J. COVID-19 vaccine tracker. 2021 [Internet. Accessed May 17, 2021]. Available from: www.raps.org/news-and-articles/ news-articles/2020/3/covid-19-vaccine-tracker

18. Boutron I, Chaimani A, Meerpohl JJ, Hróbjartsson A, Devane D, Rada G, et al. The COVID-NMA project: building an evidence ecosystem for the COVID-19 pandemic. Ann Intern Med 2020;173:1015-7.

19. Boutron I, Chaimani A, Devane D, Meerpohl JJ, Rada G, Hróbjartsson A, et al. Interventions for the prevention and treatment of COVID-19: a living mapping of research and living network meta-analysis. Cochrane Database Syst Rev 2020;11:CD013769.

20. Balshem H, Helfand M, Schünemann HJ, Oxman AD, Kunz R, Brozek J, et al. GRADE guidelines: 3 . Rating the quality of evidence. J Clin Epidemiol 2011;64:401-6.

21. Andrews JC, Schünemann HJ, Oxman AD, Pottie K, Meerpohl JJ, Coello PA, et al. GRADE guidelines: 15 . Going from evidence to recommendation-determinants of a recommendation's direction and strength. J Clin Epidemiol 2013;66:726-35.

22. Guyatt GH, Oxman AD, Kunz R, Falck-Ytter Y, Vist GE, Liberati A, et al. Going from evidence to recommendations. BMJ 2008;336:1049-51.

23. COVID19 Recommendations and Gateway to Contextualization. [Internet. Accessed May 17, 2021]. Available from: https://covid19. evidenceprime.ca

24. Geisen UM, Berner DK, Tran F, Sümbül M, Vullriede L, Ciripoi $\mathrm{M}$, et al. Immunogenicity and safety of anti-SARS-CoV-2 mRNA vaccines in patients with chronic inflammatory conditions and immunosuppressive therapy in a monocentric cohort. Ann Rheum Dis 2021 March 21 (E-pub ahead of print).

25. Boyarsky BJ, Ruddy JA, Connolly CM, Ou MT, Werbel WA, Garonzik-Wang JM, et al. Antibody response to a single dose of SARS-CoV-2 mRNA vaccine in patients with rheumatic and musculoskeletal diseases. Ann Rheum Dis 2021 March 23 (E-pub ahead of print).

26. Scully M, Singh D, Lown R, Poles A, Solomon T, Levi M, et al. Pathologic antibodies to platelet factor 4 after ChAdOx1 nCoV-19 vaccination. N Engl J Med 2021 April 16([E-pub ahead of print).

27. Schultz NH, Sørvoll IH, Michelsen AE, Munthe LA, Lund-Johansen F, Ahlen MT, et al. Thrombosis and thrombocytopenia after ChAdOx $1 \mathrm{nCoV}-19$ vaccination. $\mathrm{N}$ Engl J Med 2021 April 9 (E-pub ahead of print).

28. Greinacher A, Thiele T, Warkentin TE, Weisser K, Kyrle PA, Eichinger S. Thrombotic thrombocytopenia after ChAdOx1 nCoV-19 vaccination. N Engl J Med 2021 April 9 (E-pub ahead of print).
29. See I, Su JR, Lale A, Woo EJ, Guh AY, Shimabukuro TT, et al. US case reports of cerebral venous sinus thrombosis with thrombocytopenia after Ad26.COV2.S vaccination, March 2 to April 21, 2021. JAMA 2021 April 30 (E-pub ahead of print).

30. Government of Canada. Recommendations on the use of COVID-19 vaccines. [Internet. Accessed May 17, 2021]. Available from: www.canada.ca/en/public-health/services/immunization/ national-advisory-committee-on-immunization-naci/ recommendations-use-covid-19-vaccines.html

31. Hurd K, Barnabe C. Systematic review of rheumatic disease phenotypes and outcomes in the Indigenous populations of Canada, the USA, Australia and New Zealand. Rheumatol Int 2017; 37:503-21.

32. Barnabe C. Disparities in rheumatoid arthritis care and health service solutions to equity. Rheum Dis Clin North Am 2020;46:685-92.

33. Guyatt GH, Oxman AD, Kunz R, Woodcock J, Brozek J, Helfand M, et al. GRADE guidelines: 8. Rating the quality of evidenceindirectness. J Clin Epidemiol 2011;64:1303-10.

34. Choi MY, Barnabe C, Barber CE, Bykerk V, Pope JE, Hazlewood GS. Pragmaticism of randomized controlled trials of biologic treatment with methotrexate in rheumatoid arthritis: a systematic review. Arthritis Care Res 2019;71:620-8.

35. Monti S, Grosso V, Todoerti M, Caporali R. Randomized controlled trials and real-world data: differences and similarities to untangle literature data. Rheumatology 2018;57 Suppl 7:vii54-8.

36. Welch VA, Akl EA, Pottie K, Ansari MT, Briel M, Christensen R, et al. GRADE equity guidelines 3 : Considering health equity in grade guideline development: rating the certainty of synthesized evidence. J Clin Epidemiol 2017;90:76-83.

37. Feldman $\mathrm{CH}$, Ramsey-Goldman R. Widening disparities among patients with rheumatic diseases in the COVID-19 era: an urgent call to action. Arthritis Rheumatol 2020;72:1409-11.

38. Mehta B, Jannat-Khah D, Fontana MA, Moezinia CJ, Mancuso CA, Bass AR, et al. Impact of COVID-19 on vulnerable patients with rheumatic disease: results of a worldwide survey. RMD Open 2020;6:e001378.

39. Michaud K, Wipfler K, Shaw Y, Simon TA, Cornish A, England $B R$, et al. Experiences of patients with rheumatic diseases in the United States during early days of the COVID-19 pandemic. ACR Open Rheumatol 2020;2:335-43.

40. U.S. Food \& Drug Administration. FDA Briefing Document. Janssen Ad26.COV2.S vaccine for the prevention of covid-19. Vaccines and Related Biological Products Advisory Committee Meeting, February 26, 2021. [Internet. Accessed May 25, 2021.] Available from: www.fda.gov/media/146217/download 